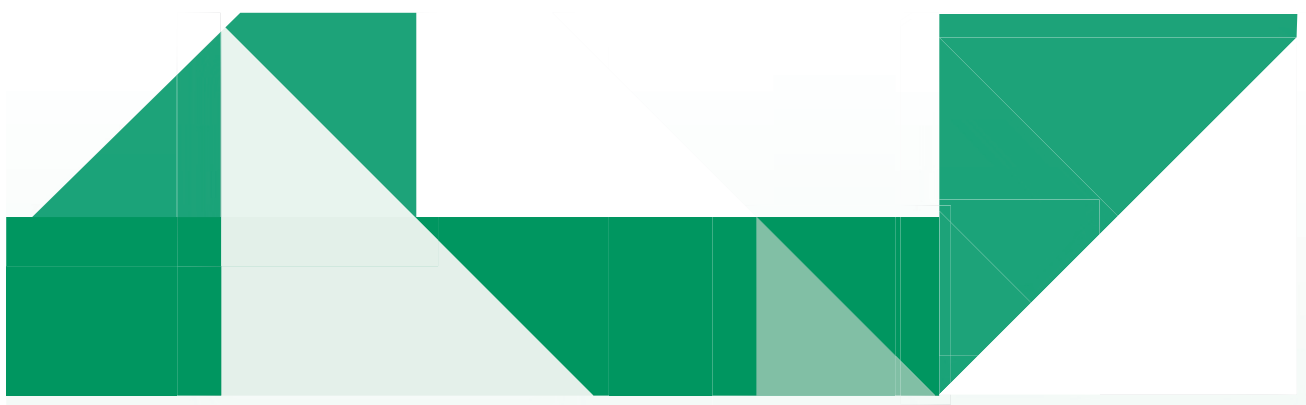

\title{
1) Teoría de la integración conceptual: una mirada desde la interacción con un robot móvil
}

- Teoria da integração conceitual : a vista a partir da interação com um robô móvel

- The conceptual integration theory: an approach from the interaction with a mobile robot

\section{Resumen}

En esta investigación se pretende analizar los procesos de significación que se presentan en la interacción que tienen las personas con los objetos tecnológicos. En este caso se analiza el resultado de la interacción entre un niño y un robot móvil controlado con un dispositivo que suprime el contacto físico a partir de la implementación de una cámara RGB y un sensor de profundidad, llamado Kinect, diseñado por Microsoft. Este estudio se analiza teniendo como referente la teoría de la integración conceptual planteada por Fauconnier y Turner; la cual busca entender cómo emergen nuevas ideas a partir del reconocimiento y la integración de elementos preexistentes. La anterior teoría nace de los postulados de Lakoff y Johnson, de sus estudios sobre la construcción de sentido en la metáfora, para que luego esta sea utilizada en el campo de la semiótica.

El presente trabajo es de corte cualitativo e involucró a miembros de una institución educativa de la ciudad de Bogotá, quienes interactuaron con un robot móvil tratando de conducirlo de un lugar a otro, usando como interfaz el dispositivo Kinect. Estas experiencias fueron documentadas a través de videos y entrevistas, en las cuales se indagaba sobre la proyección del ejercicio desde escenas previas y hacia nuevos escenarios. Los análisis realizados a la luz de la teoría de la integración conceptual dieron cuenta de las relaciones cognitivas que se presentaban. No obstante las diferencias generacionales de los participantes, se establecen elementos en común, los cuales se observan la formulación y construcción de la red de integración conceptual.

Los hallazgos, en general, permiten establecer que en el proceso de significación que se presenta al interactuar con un objeto nuevo se requiere de experiencias previas con objetos ya establecidos y definidos por una cultura. En ese sentido, cobra vigencia el concepto de anclaje material planteado por Hutchins, para entender lo que sucede a nivel del cambio de frame cultural. Asimismo dichos anclajes materiales, se presume, pueden llegar a convertirse en insumos de nuevas redes conceptuales. De acuerdo con esta premisa es posible contemplar

Magíster en Tecnologías de la Información Aplicadas a la Educación y licenciado en Diseño Tecnológico de la Universidad Pedagógica Nacional. Profesor de la Escuela Tecnológica Instituto Técnico Central y de la Corporación Unificada Nacional (CUN). Correo electrónico: roycano78@gmail.com.ORCID: orcid. org/0000-0002-7944-563X.

** Magíster en Semiótica de la Universidad Jorge Tadeo Lozano y licenciado en Diseño Tecnológico de la Universidad Pedagógica Nacional. Profesor de la Secretaría de Educación Nacional y de la Corporación Universitaria Minuto de Dios (Uniminuto). Correo electrónico: rcamilot@gmail.com. ORCID.org/0000-0001-9333-5747.

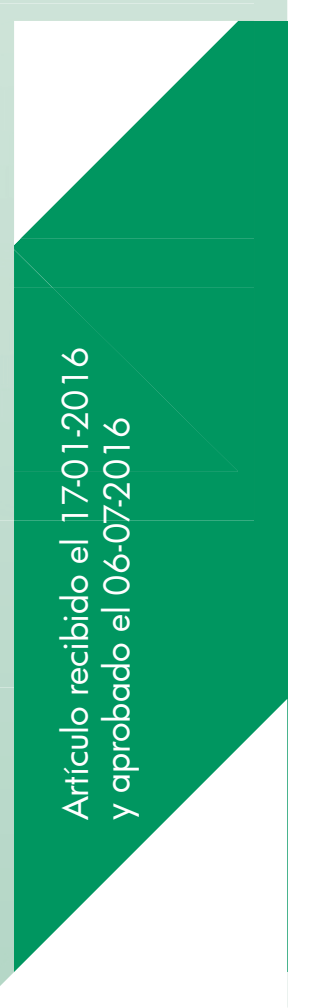


la formulación de nuevas metodologías de aprendizaje que conduzcan a obtener mejores resultados en el contexto escolar.

Palabras clave

Teoría de la integración conceptual, espacios mentales, robótica educativa, metodología de aprendizaje, diseño

\section{Resumo}

Esta pesquisa pretende analisar os processos de significação que se apresentam na interação que têm as pessoas com os objetos tecnológicos. Neste caso foi analisado o resultado da interação entre uma criança e um robô móvel controlado com um dispositivo que elimina o contato físico a partir da implementação de uma câmera RGB e um sensor de profundidade, chamado Kinect, desenvolvido pela Microsoft. A análise deste estudo tem ponto de partida na teoria da integração conceitual representada pelo Fauconnier \& Turner; a qual procura entender como emergem as novas ideias a partir do reconhecimento e a integração dos elementos preexistentes. Os postulados da teoria anterior nascem dos postulados de Lakoff e Johnson, dos estudos sobre a construção do sentido na metáfora, para que logo está seja utilizada no campo da semiótica.

O trabalho apresentado é de ordem qualitativa e envolveu membros de uma instituição de ensino na cidade de Bogotá, os quais quem interatuaram com um robô móvel, tratando de dirigi-o de um lugar para o outro usando como Interface o sensor Kinect. Estas experiências foram documentadas através de vídeos e entrevistas, nas quais se indagou a projeção que tinha o exercício das cenas previas para os novos cenários. As análises realizadas à luz da teoria da integração conceitual mostraram as relações cognitivas que se apresentavam. Porém as diferenças geracionais dos participantes, se estabeleceram elementos em comum, os quais se observam na formulação e construção da rede de integração conceitual.

Os achados, em geral, permitem estabelecer que o processo de significação que se apresenta ao interagir com um novo objeto, é necessário possuir experiência com objetos já estabelecidos e definidos por uma cultura. Nesse sentido, o conceito de vinculo material (anclaje) torna-se relevante, o exposto por Hutchins, para entender o que acontece ao nível de mudança de marco cultural. Da mesma forma tais vínculos (anclajes) materiais presumivelmente, podem-se tornar insumos para novas redes conceituais, sob esta premissa é possível prever o desenvolvimento de novas metodologias da aprendizagem que levam a melhores resultados no contexto escolar.

Palavras chave:

teoria da integração conceitual, espaços mentais, robótica educativa, metodologia de aprendizagem, desenho

\section{Abstract}

This research aims to analyze the processes of meaning that arise in the interaction between people and technological objects. In this case, we analyze the results of the interaction of a child with a mobile robot controlled by a device that suppresses the physical contact by a RGB camera and a deep sensor (Kinect by Microsoft). The reference for the study is the Conceptual Integration Theory proposed by Fauconnier and Turner, which seeks to understand how new ideas are constructed from appreciation and integration of pre-existing elements. Their foundations are Lakoff and Johnson's postulates, which deal with the sense-building process in metaphors in order to use it in the field of semiotics.

This is a qualitative study which involved some participants from an educational institution in Bogota. They interacted with a mobile robot trying to move it from one place to another by using the Kinect device. The experiences were documented by using tapes and interviews in which participants were asked about the exercise projection from the old scenes to the new ones. The analysis carried out in the light of the conceptual integration theory showed the emergent cognitive relationships. In spite 
of generational differences of participants we could notice common elements, which are observed on construction and formulation of conceptual integration network.

The findings allow the researchers to conclude that during the meaning process, which appears in the interaction with a new object, we need previous experiences with pre-stablished objects defined by a culture. Additionally Hutchins material anchor is important to understand what is happening at the cultural frame change level. Likewise those material anchors, we assumed, could turn in a new input for conceptual networks. Thus it is possible to look at the formulation of new learning methodologies in order to obtain better results in the academic field.

Keywords

Theory of Conceptual Integration, mental space, educational robotics, learning methodology, design 


\section{Introducción}

En la actualidad los robots se han convertido en elementos cotidianos para desarrollar distintas actividades, entre ellas: el entretenimiento, la seguridad, la industria y la educación. La robótica educativa como estrategia didáctica representa una oportunidad para llevar a los estudiantes a concebir, diseñar y desarrollar dispositivos que les permitan abordar conceptos científicos y tecnológicos para la solución de problemas (Ruiz-Velazco, 2007).

Habitualmente los robots son programados para la realización de tareas específicas que son supervisadas por humanos o son controlados a distancia por medios tangibles. De allí surge un cuestionamiento sobre si la relación será distinta al modificar la forma de control involucrando tecnologías que suprimen el contacto directo, como el sensor Kinect.

La iniciativa de investigación nace de las experiencias previas tenidas en la interacción con videojuegos mediados por la tecnología Kinect, que se caracterizan por la presencia de interfaces virtuales, que transportan a las personas a otras realidades. Ahora bien, genera interés lo que sucede a nivel cognitivo cuando las personas interactúan con objetos tangibles controlados con Kinect, para este estudio un robot móvil.

Este fenómeno se pretende explicar con base en la teoría de la integración conceptual, propuesta por Fauconnier y Turner (2002), la cual busca entender el proceso de construcción de significado a partir de estructuras de pensamiento conocidas como espacios mentales. La elección de la teoría obedece a la posibilidad de observar el proceso de construcción de un significado nuevo a partir de la integración de paquetes de conceptos.

\section{La teoría de la integración conceptual en la interacción con los objetos}

La teoría de los espacios mentales (TEM), fue propuesta por Fauconnier (1994) como una forma de responder a problemas en torno a la referencia. Posteriormente esta teoría fue ampliada por el mismo Fauconnier pero en compañía de Turner (2002), y esta extensión es conocida como la teoría de la integración conceptual (TıC).

Para comprender algunos de los aspectos que se abordaran en el siguiente documento es oportuno hacer ciertas precisiones. Un componente primordial lo constituyen los espacios mentales, que son "pequeños paquetes de conceptos construidos como nosotros pensamos y hablamos, para propósitos de comprensión local y acción" (Fauconnier y Turner, 2002, p.40). Estos se generan y modifican en la medida en que se desarrollan el pensamiento y el discurso y se conectan los unos con los otros por varias clases de mapeos, es decir, proyecciones entre los elementos y las relaciones de un espacio a otro. 
En un espacio mental asisten de forma temporal diferentes tipos de información que provienen, por ejemplo, de lo que acabamos de decir o percibir, del entorno, del medioambiente o de la memoria a largo plazo. Cuando estos elementos se relacionan y se organizan en un paquete que ya conocemos, decimos que el espacio está enmarcado y llamamos a esa organización un frame'. Reconocidos como "conectores esquemáticos de conocimiento a largo plazo" (Turner, 2002, p. 40), se establecen de dos maneras: abstractos y organizadores, en aras de la comprensión se revisará un ejemplo citado por Niño (2015), referido al de "competencia y pelea de boxeo, el primero no es organizador, debido a que se refiere a una generalidad, mientras el segundo sí lo es, esto se debe a la manejabilidad y la relevancia cognitiva" (p. 264).

Ahora bien, tal como se mencionó anteriormente, la TEM dio origen a la TIC, que tiene como idea central la construcción de significado a partir de la integración de estructuras -las cuales deben ser vistas no como la suma de sus partes, sino como la generación de una red integrada-denominadas redes de integración conceptual (RIC), conformadas de la siguiente manera (Fauconnier y Turner, 2002):

- Por dos o más espacios de entrada o inputs. Cada espacio de entrada se puede considerar como un espacio mental, y como tal, algo que contiene información parcial, construida en línea, y se presenta en la medida en que pensamos.

- Al menos por un espacio genérico, que captura información común a los dos espacios de entrada.

Para efectos de comprensión de la teoría de la integración conceptual, los conceptos propios no serán traducidos ya que pueden causar confusiones.
- Un espacio integrado o blend, que incorpora elementos que se han proyectado selectivamente desde los espacios de entrada.

La manera como se genera la integración, según este modelo, es a partir de mapeos parciales entre los espacios de entrada y de selección proyectiva desde los espacios de entrada hasta el blend. "La integración conceptual ocurre en el momento de la percepción y crea nuevos significados a partir de caminos existentes del pensamiento" (Joy, Sherry, Deschenes, 2007, p.41). El blend hereda parte de la estructura de los espacios de entrada, pero también presenta una estructura emergente propia que se obtiene por"composición y compleción de patrones o modelos conceptuales disponibles" (Fauconnier y Turner, 2002, p.47). La estructura emergente del blend va a permitir, además, hacer la elaboración, esto es, hacer "inferencias o extraer consecuencias con base en la información que se ha reestructurado a partir de los otros procesos" (Ibíd, 2002, p.40), en otras palabras hacer correr el blend. Es decir, se proponen tres momentos para el proceso de integración: composición, compleción y elaboración. No es necesario suponer que hay una secuencia temporal en la construcción de las RIC, pues todos esos procesos pueden suceder simultáneamente.

Para comprender mejor lo mencionado, es preciso presentar dos ejemplos que ilustrarán cómo se generan las RIC. El primero aborda la manera como posiblemente se concretó el diseño de un portaminas. Éste nace del lápiz, el cual se concibe como un objeto, representativo de la capacidad del hombre para abstraer sus pensamientos, aislarlos o reproducirlos -icónica o lingüísticamente- enla superficie de un papel. Este objeto está conformado por un alma de grafito, un cuerpo de madera hexagonal y un cierre en la punta, el borrador. Con el grafito se pueden realizar marcas -escribir o 
dibujar-que se pueden borrar, es decir, se eliminan o se cancelan. En contraste con la pluma o el esfero, el lápiz permite la corrección en el papel, característica fundamental de este objeto, pero con un inconveniente: hay que afilarlo, renovar siempre el grafito, retirarle su cuerpo de madera, esto hace que este llegue a un fin. El inventor japonés Tokuji Hayakawa propuso una manera de superar tal dificultad: diseñó un dispositivo mecánico que expulsaba una mina interna alojada en un cuerpo metálico y lo denominó "lápiz de punta eterna", es decir que nunca pierde el filo.

Entender que un lápiz tiene una punta finita permite el contrafáctico ${ }^{2}$ de punta eterna, que en palabras de Markusseen y Krogh (2008) es un cambio de frame cultural, representado en la figura 1 :

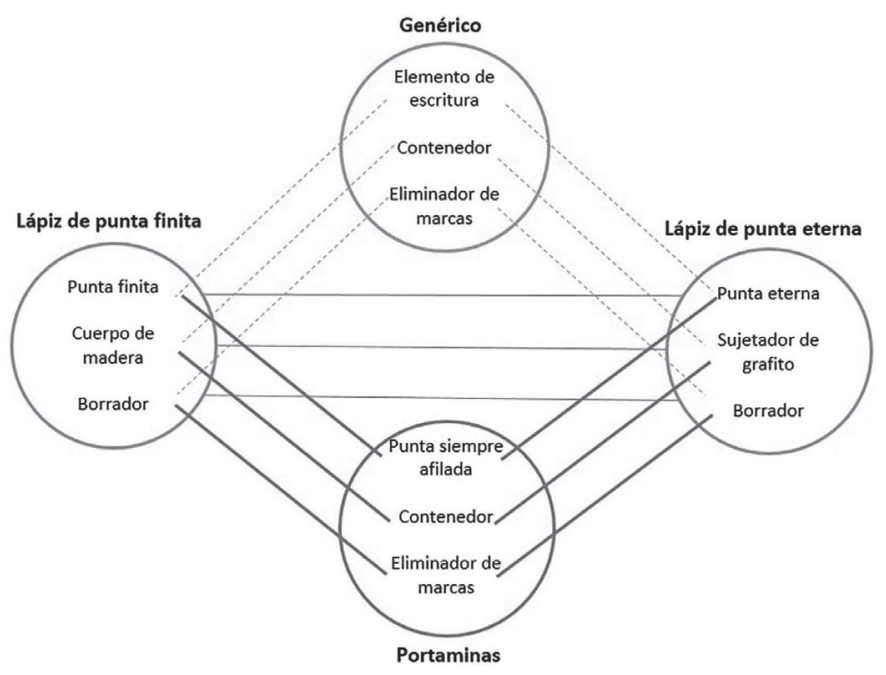

Figura 1. RIC portaminas

- Input 1 : Elementos: "Punta finita", "Cuerpo de madera" y "Borrador". Relaciones: "Realizar marcas (escribir o dibujar)" y "Borrar". Esta información se recluta en el frame "[Lápiz de punta finita]", en donde se incluye el rol realizar marcas que se pueden corregir en el papel.

- Input 2 : Elementos: "Punta eterna", "Sujetador de grafito" y "Borrador". Relaciones: "Realizar marcas (escribir o dibujar)" y "Eliminar". Esta información se reúne en el frame "[Lápiz de punta eterna]", en donde se incluye el rol realizar marcas que se pueden corregir en el papel.

- Genérico: "Grafito", "Contenedor" y "Borrador".

- Blend: "Punta finita" y "Punta eterna" => "Punta siempre afilada"; "Cuerpo de madera" y "Sujetador de grafito" => "Contenedor"; "Borrador" $\mathrm{y}$ "Borrador" => "Borrador"; estos elementos se agrupan en el frame "[Portaminas]", generado a partir de los rasgos enmarcados en la

2 Los contrafácticos se conocen como enunciados condicionales contrarios a los hechos, incumplidos o subjetivos. 
similaridad, debido a las propiedades compartidas, igualmente a que el rol que va a desempeñar este objeto es el de dejar marcas borrables en un papel.

En este ejemplo se puede observar que la integración conceptual se da a partir de una idea y un objeto de uso, que es un ancla material (Hutchins, 2005). Es decir, lo que se presenta es una asociación entre una estructura conceptual y una estructura material. La creación del lápiz de punta eterna se presenta a partir de una disanalogía, debido a que lo que se buscaba con la creación de este objeto no era sacar de afuera hacia dentro el grafito, sino generar un dispositivo que realizara todo lo contrario, lo que se busca con este objeto es desplazar la mina, sin que exista ningún desgaste del contenedor, eliminando el uso de un afilador.

Para entender de manera más clara el blend que se presenta acá, analizaremos los procesos de la integración: composición, compleción y elaboración. La composición para este caso consiste en imaginar una punta eterna en un cuerpo de madera; lo que se observa es que el blend gracias a la proyección selectiva que se realiza desde los inputs adopta relaciones que no existen en los inputs por separado. Es importante darse cuenta de que en la proyección selectiva de los elementos y las relaciones presentes en los inputs, algunas cosas se proyectan y otras no.

La compleción en el ejemplo consiste en traer dos ideas opuestas, la punta finita frente a la punta eterna, un contra fáctico, con lo cual se modifica el frame cultural y se hace visible el desarrollo de la creatividad para ir más allá de lo explícito. Aquí se presenta una reacomodación o ajuste de la estructura emergente que aparece en la composición, lo cual puede traer a colación información del frame
- de los frames en cuestión, a partir de la memoria a largo plazo.

Finalmente, se encuentra la elaboración, que consiste en la simulación mental o física de la ejecución del blend, teniendo en cuenta su propia estructura emergente. Esto permite "correr el blend", para extraer consecuencias, hacer inferencias, anticipar efectos. Para el caso del ejemplo, se trata de inferir un lápiz el cual siempre se encontrará afilado y cuyo contenedor nunca finalizará; a la vez permitirá entender que se necesita un mecanismo que empuje la mina y que el portaminas es un objeto recargable.

Como segundo ejemplo se expone la experiencia relacionada con la interacción con el videojuego PacMan, familiar para la mayoría de generaciones recientes. Su éxito radica en la simplicidad del argumento que esconde el juego, de los pocos personajes que lo integran, de las emociones que produce y de su jugabilidad. El juego transcurre en escenarios en forma de laberinto, donde el personaje principal es perseguido por unos fantasmas, mientras intenta comer la totalidad de galletas (puntos) que aparecen en el camino. La significación producida por este juego se puede entender en la RIC de la figura 2 :

Elementos de la red de integración conceptual:

- Input 1: Elementos: "Ladrón", "Bienes ajenos" y "Policías". Relaciones: "Rol/ Valor", inherentes a cada personaje. Los cuales se articulan al frame: "[Policías y ladrones]".

- Input 2 : Elementos: "PacMan", "Galletas" y "Fantasmas". Relaciones: "Rol/ valor" enmarcados en las funciones de huir y perseguir. Elementos articulados en el frame: "[Videojuego PacMan]". 


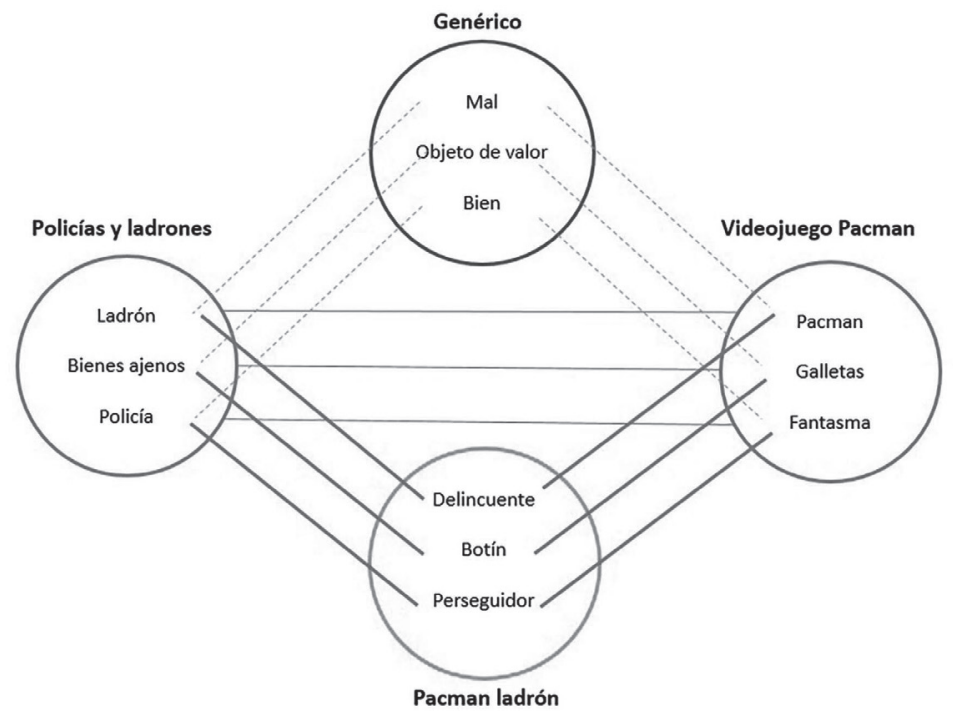

Figura 2 RIC videojuego Pacman

- Genérico: "Mal", "Objeto de valor" y "Bien".

- Blend: Elementos: "Ladrón" y "PacMan" => "Delincuente"; "Bienes ajenos" y "Galletas" => "Botín"; "Policía" y "Fantasma" => "Perseguidor"; estos elementos se agrupan en el frame: "[PacMan ladrón]".

Los espacios de entrada tienen elementos en común que son mapeados entre sí, es decir, "[Policías y ladrones]" cuenta con tres elementos: ladrón, bienes ajenos y policía. "[Videojuego PacMan]" cuenta con tres elementos homólogos: PacMan, galletas y fantasmas. Por otro lado, observamos que la construcción del espacio genérico revela tres elementos proyectados: mal, objeto de valor y bien. Precisamente, allí se evidencia una categorización de los elementos al inscribirlos en un concepto más general que los abarque.

La integración da como resultado el espacio "[PacMan ladrón]", que recoge elementos de los espacios de entrada representados endelincuente, que resume las características de ladrón y PacMan, se configura en razón a la actividad delictiva de comerse los recursos que no le pertenecen. Botín adopta el rol y las propiedades de los elementos bienes ajenos y galletas, y se convierte por lo tanto en el objetivo central que se pretende alcanzar para superar cada nivel del juego. Por último, perseguidor representa las proyecciones de policía y fantasma, ya que estos elementos heredan el rol y la propiedad que les permiten ir detrás del personaje para evitar el robo o capturar al ladrón.

Para entender mejor la integración que se da en el ejemplo anterior, es preciso revisar el tipo de relación vital (Fauconnier y Turner, 2001), que explica el tipo de asociación presente entre los elementos de los espacios mentales de entrada, se anota que existen varias relaciones. Para el caso mencionado tenemos que la de rol/valor y propiedad; la primera derivada por la función y actividad que realizan; 
y la segunda, porque se presentan elementos que heredan características de los espacios predecesores. Los policías persiguen a los ladrones y los fantasmas persiguen a PacMan.

Así mismo, podemos decir que las relaciones provenientes de los espacios mentales de entrada y del espacio genérico son claras y consistentes, sustentadas en la construcción del espacio integrado "[PacMan ladrón]" como una unidad manipulable con estructura definida e interrelacionada. Además, no se pierden las referencias a los espacios mentales de entrada, ya que se puede mantener la independencia de aquellos para participar en la construcción de otras integraciones. Por estos argumentos los principios de integración, red, topología, desempaquetamiento y relevancia estarían cubiertos.

\section{Especificidades del robot móvil}

Para entender la manera como la TIC está presente en la interacción entre una persona y un robot móvil es preciso detallar las características que acompañan dicho objeto y el medio en el cual se dio tal experiencia. La función principal del robot es proyectar los movimientos corporales de quien lo controla, a diferencia de lo que ocurre en un proceso de imitación en el que existe la voluntad del otro individuo para producir la acción, imaginemos la actuación de un mimo.

Ahora bien, el dispositivo diseñado acoge los elementos funcionales de cualquier robot móvil e integra aspectos de usabilidad. Para permitir su desplazamiento, el robot cuenta con dos motores, un elemento de control y administración del flujo de información, que para este caso es la placa de programación Arduino ${ }^{3}$, una tarjeta bluetooth que transfiere

3 Arduino es una plataforma electrónica de recurso abierto basada en el uso fácil del software y el hardware. Está diseñada para realizar proyectos interactivos. los datos inalámbricamente y una batería de alimentación. Por otra parte, se utiliza como interfaz de comunicación entre el usuario y la máquina un Kinect, recurso propio del ámbito de los videojuegos, novedoso por la posibilidad que le da al usuario de controlar las acciones sin contacto directo con la máquina. Asimismo, se aprovecha la facilidad de programación que brinda el ambiente Scratch ${ }^{4}$ para la materialización de las instrucciones que surgen desde la persona que interactúa con el objeto.

\section{Interacción con el robot móvil}

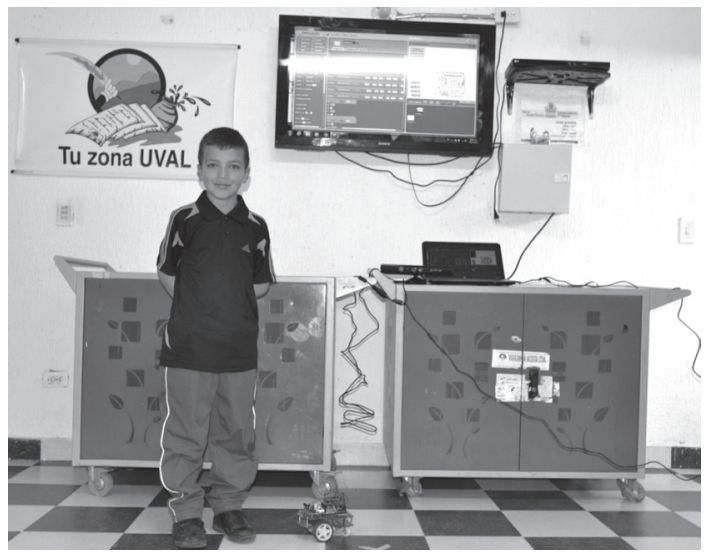

Fotografía 1 Interacción con el robot

[Fotografía de Óscar Ramírez]. (Bogotá, 2015). Colección personal.

El lugar donde ocurre la experiencia es el colegio rural El Uval, localizado en la localidad de Usme de la ciudad de Bogotá. La formación que imparte es educación básica y media. La población que atiende mayoritariamente es de bajos recursos económicos y existen problemáticas de tipo social.

Los interrogantes que esta investigación desarrolla se refieren a: ¿̇qué sucede a nivel

4 Scratch es un entorno de aprendizaje de lenguaje de programación, que permite a los principiantes aprender a escribir de manera sintácticamente correcta primero. Permite investigar, introducirse y jugar con la programación de ordenadores utilizando una interfaz gráfica muy sencilla. 
cognitivo desde el punto de vista de la TIC, cuando una persona interactúa con un robot móvil controlado con Kinect? ¿Qué utilidad representa la TIC en campos como la educación o el diseño? La solución a estas preguntas se abordará a través de un estudio de caso interpretativo, caracterizado por:

Aportar descripciones densas y ricas con el propósito de interpretar y teorizar sobre el caso. El modelo de análisis es inductivo para desarrollar categorías conceptuales que ilustren, ratifique o desafíen presupuestos teóricos difundidos antes de la obtención de la información. (Barrio et al., 2015, p. 4)

La planeación de la experiencia giró en torno a un reto que consistía en posicionar el robot en tres puntos determinados, marcados en el suelo con tres señales que denotaban el origen, un punto intermedio y el final. La solución de este reto se dio con la interacción con el robot móvil; para ello se le pidió a la persona que hiciera desplazar el dispositivo sin ningún tipo de instrucción previa. Posteriormente se realizó una entrevista a los actores involucrados en este proceso para que narraran su experiencia, basados en unas preguntas guía. En este ejercicio se contó con la participación de tres estudiantes, dos niñas y un niño con edades entre los 8 y 9 años, y a la vez, un docente.

En un inicio, la atención por parte de los involucrados se centró en la identificación de los componentes que integran el dispositivo móvil, reconociendo el robot, el Kinect y el reto por solucionar. Luego exploraron las diferentes posibilidades que les brinda su cuerpo para controlar el robot, haciendo uso de su cabeza, sus extremidades, etc. Sin embargo se evidenciaron dificultades para establecer un adecuado control, por lo que se aprovechó para dar pautas que afinaran el dominio del robot, se comentó que era preciso hacer uso de los miembros superiores e inferiores, y asociarlos a la lateralidad. Con la pretensión de maximizar la oportunidad de manipular naturalmente el dispositivo, y a la vez, de hacer uso de ciertos mecanismos de proyección corporal. Al familiarizarse con los movimientos que permitían que este objeto se desplazara, paulatinamente fueron mejorando sus estrategias para cumplir con el reto.

Para finalizar, se procedió a dialogar con los estudiantes y el docente sobre los detalles que enmarcaron la experiencia. La charla sostenida aquí giró en torno a unas preguntas que pretendían indagar sobre las integraciones conceptuales realizadas. Estas eran abiertas, y con ellas se buscaba que las personas narraran con naturalidad lo vivido, evitando inducirlos a respuestas deseadas.

\section{La teoría en funcionamiento}

Durante la interacción que realizaron los niños con el dispositivo, se observaron algunas coincidencias de su comportamiento, las cuales vale traer a colación, debido a que permitirán entender cómo la Tic se encuentra presente en este ámbito.

Una característica fundamental es que sus movimientos tratan de sugerir la trayectoria que desean que siga el robot. Aunque los niños nunca entran en 
contacto directo con el objeto, se identifica que la mano está en posición de agarre, su brazo se encuentra extendido y el movimiento que realiza es de empujar. Sus piernas alternadamente aparecen extendidas para describir una trayectoria lineal hacia uno de los costados. Las anteriores dos acciones poseen una fuerza elevada, tensión corporal marcada y uso de energía considerable, porque los participantes tienen en cuenta las propiedades físicas inherentes al robot, como son su peso y volumen.

A pesar de que los niños tuvieron en cuenta que para controlar el robot requerían de sus extremidades, predominó el uso de su lado diestro, desestimando las posibilidades de dominio que se tenían al utilizar en conjunto sus brazos y piernas, lo cual dificultó la solución del reto que se les formuló en un principio.

Cabe aclarar que la manera como fue concebida la programación del robot en relación con su movimiento adoptaba conceptos de lateralidad, es decir, que para girar a la derecha o izquierda se deberían desplegar los brazos, alejándolos de su plano sagital. De la misma forma, para desplazar el robot hacia delante o atrás, era necesario subir la pierna derecha o izquierda respectivamente.

Para el caso de la interacción del adulto se reconocen algunos elementos para analizar, que al contrario de lo sucedido con los niños, el profesor se identificó con la mecánica de funcionamiento, evitando estrategias de ensayo-error y reconociendo que sus movimientos desencadenan un efecto en el dispositivo. Sus actuaciones dejan ver que existen experiencias previas con el uso de la interfaz Kinect, lo que se puede interpretar, a la luz de la teoría, como un atrincheramiento de conceptos evocados desde su memoria de largo plazo. La tensión, energía y fuerza utilizadas fueron menores que las de los niños, aparentemente por su acercamiento al tema de los videojuegos que usan esta tecnología; dominó el ejercicio en poco tiempo y tuvo la posibilidad de afinar sus estrategias de operación.

Las acciones de control relacionadas con los movimientos hacia delante y atrás del robot, coincidieron con lo presupuestado en el algoritmo de programación, como se observa en el uso de las piernas, al subirlas o bajarlas, para activar el desplazamiento. Asimismo, un aspecto diferencial con los otros participantes es el hecho de que el adulto encontró elementos de proporcionalidad al identificar que la velocidad aumentaba directamente al elevar su brazo o sus piernas.

Como se mencionó, las acciones que realizaron los participantes dejan ver que su cuerpo es protagonista en la meta de controlar los movimientos de un robot con el que no se encuentran en contacto directo. En ese sentido, la TIC permite dilucidar en la experiencia vivida anteriormente elementos de cambio a nivel de frame cultural, reconocer anclajes materiales, facilitar la construcción de significados y apoyar los procesos de diseño. En cumplimiento de tales objetivos, el uso del modelo de la red de integración conceptual (RIC) faculta a través de un esquema la representación de los elementos que intervienen en la interacción.

Una parte del sentido que emerge del proceso de interacción ya detallado, corresponde a la presencia del cuerpo como control remoto. Para entenderlo es preciso identificar cuáles son los elementos que dan origen a esa integración, mostrados en la figura 3 : 


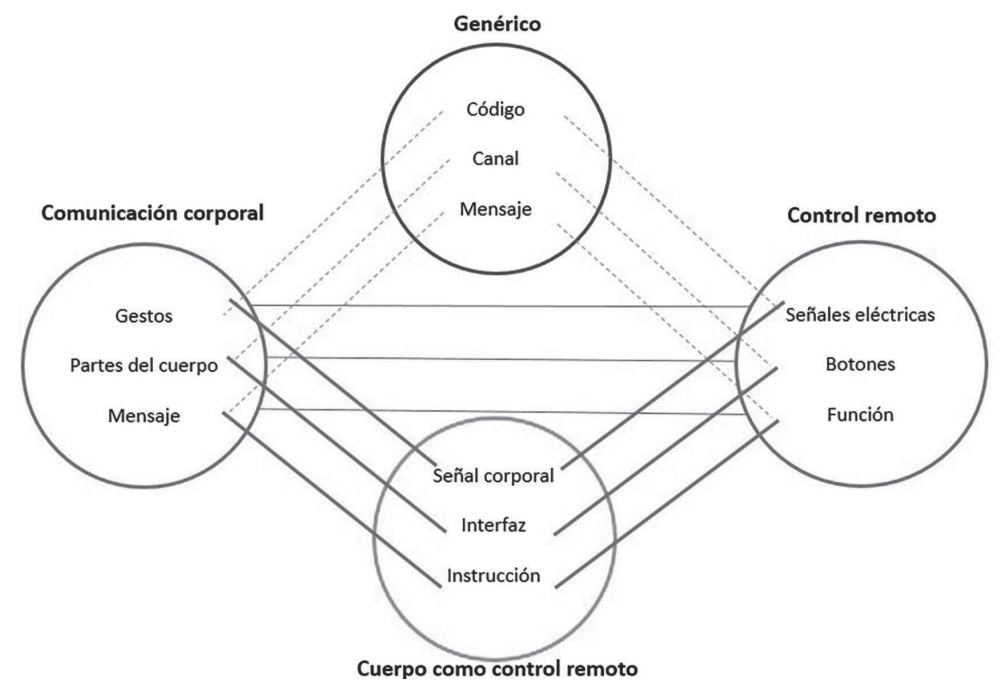

Figura 3 RIC cuerpo como control remoto

- Input ${ }_{1}$ : Elementos: "Gestos", "Partes del cuerpo", "Mensaje". Relación: Rol y propiedad, por el papel que desempeña el cuerpo en la comunicación. Se articulan en el frame "[Comunicación corporal]".

- Input 2 : Elementos: "Señales eléctricas", "Botones", "Función". Relaciones: Parte al todo y propiedad por el sistema de comunicación. Se articulan en el frame "[Control remoto]".

- Genérico: Elementos: "Código", "Canal", "Mensaje".

- Blend: Elementos: "Gestos" y "Señales eléctricas" => "Señal corporal"; "Partes del cuerpo" y "Botones" => "Interfaz"; "Mensaje" y "Función" $=>$ "Instrucción". Se agrupan en el frame "[Cuerpo como control remoto]".

El espacio mental comunicación corporal se relaciona con un objeto real, que es un control remoto, que es en sí un anclaje material. Este es un mando a distancia diseñado para la interacción con una máquina, generalmente para usarlo es necesario manipularlo con las manos. Las funciones que están asignadas a cada botón del control se pueden ver reflejadas en la existencia de otro espacio mental, donde ciertas partes del cuerpo humano asumen tales funciones para controlar un dispositivo. Esto explicaría la existencia de relaciones vitales de rol y de propiedad.

La RIC ilustra que existe una secuencia de relaciones entre las características de cada espacio, recogidas en un espacio más complejo, el blend. Los ítems que emergen poseen una secuencia de relaciones y propiedades amalgamadas, entendidas como una fusión mas no como una suma de elementos. En síntesis, se observa en el blend una interdependencia entre los nuevos elementos que no permite la exclusión de ninguno de ellos sin que pierda sentido el espacio mental creado. 
El hecho de que la persona pueda controlar un dispositivo con su cuerpo no quiere decir que esta se convierte en un objeto, por el contrario conserva sus características y mantiene consciencia de sus actos. La idea de control a través de un mando tangible desaparece para dar paso al cuerpo como elemento de control, lo que implica cambios a nivel cultural que obligan a replantear la interacción con algunos objetos.

Como se observa, esta RIC explica solo una parte del sentido que se originó en la experiencia vivida, dado que la persona asume el rol de un control remoto y las propiedades del mismo, hasta el momento no es posible determinar en qué circunstancias ocurre el movimiento del objeto. Ello propicia la conformación de una RIC más elaborada que dé cuenta de cómo mover un robot desde la distancia sin la participación de otro elemento tangible.
- Input, : Elementos: "Señal corporal", "Interfaz", "Interacción". Relaciones: rol y propiedad heredadas de la construcción de la RIC anterior. Se articulan en el Frame "[Cuerpo Como Control Remoto]".

- Input 2 : Elementos: "Pulso eléctrico", "Sensor", "Posicionamiento". Relaciones: Parte al todo y propiedad por el sistema de comunicación. Se articulan en el frame "[Moverrobot]".

- Genérico: Elementos: "Código", "Medio", "Mensaje".

- Blend: Elementos: "Señal corporal" y "Pulso eléctrico" => "Señal codificada"; "Interfaz" y "Sensor" => "Mando intangible"; "Interacción" y "Posicionamiento" $=>$ "Desplazamiento". Se agrupan en el frame "[Mover objeto con el cuerpo]".

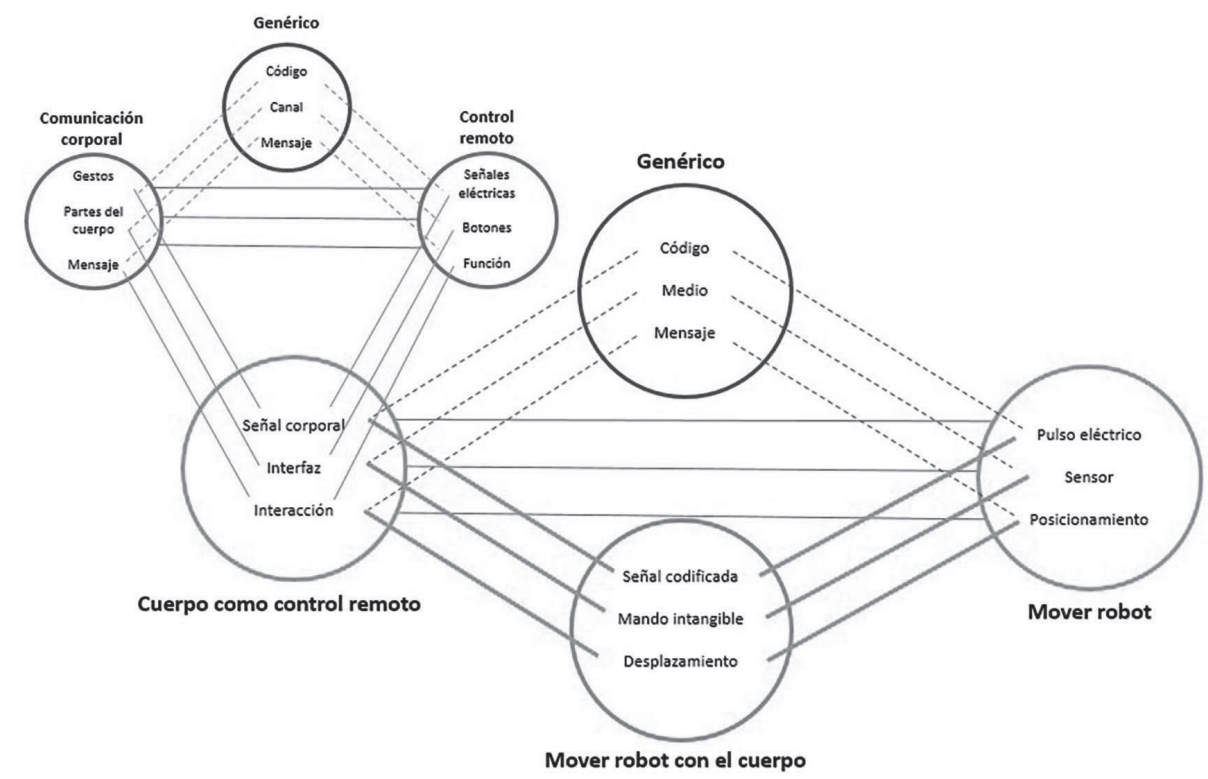

Figura 4 RIC mover robot con el cuerpo. 
De la integración conceptual podemos evidenciar que un blend se convierte en uno de los inputs de este esquema, a lo que Fauconnier y Turner llaman hiperblends (citado por Niño, 2015, p.283). Los ítems de cada nuevo input se complementan entre sí, con sus propiedades, y dan lugar al blend. Así se reafirma que la RIC discutida explica el proceso de significación que se presenta en la interacción, planteado en la postura de Malagón: el proceso de significación es más complejo de lo que parece y requiere de múltiples integraciones para generar significados coherentes. Allí radica la explicación sobre el hecho de que haya personas que lleven a cabo "procesos de significación acertados o no" (2013, p. 179). En ese sentido, las características del frame "[Cuerpo como control remoto]", necesitan de un complemento, en este caso proporcionado por el input del frame "[Mover robot]", que facilita el desplazamiento. La convergencia de estos espacios mentales con sus propiedades es lo que permite el alcance del objetivo de controlar el robot representado en el blend del frame "[Mover robot con el cuerpo]". Dentro de la TIC, esto corresponde al proceso de composición.

La compleción, específicamente, la podemos enunciar en la acción de jugar, la cual no fue explícita en ninguno de los participantes; sin embargo, se asume que la presencia de recursos como el Kinect, el ambiente de programación y el robot motivan a que las personas asocien la actividad con un juego. De igual forma, las mismas características que permitieron definir el frame "[Control remoto]" como un objeto real aceptan la existencia de nuevas condiciones relacionadas con la presencia de un control virtual, reafirmado en el frame "[Cuerpo como control]".

Finalmente, la elaboración permite entender los argumentos expuestos por los participantes en los que mencionaban que dicha tecnología puede ser utilizada en otros contextos, como en su hogar, el transporte de objetos o en labores artísticas, como pintar murales. Se da a entender que las entradas al blend son una representación mental de una estructura material, en sí una representación mental de los elementos conceptuales presentes (Hutchins, 2005).

Aunque es difícil definir las fronteras que delimitan las relaciones vitales, debido a que se presentan al mismo tiempo y durante el proceso de interacción, sí es posible identificar algunas de ellas: causa-efecto, rol-valor, propiedad y proyección. La relación causa-efecto se da en las dinámicas de funcionamiento del robot, ya que al realizar una acción con el cuerpo, esta tiene una repercusión en la activación del dispositivo. Rol-valor, esta relación es heredada de los espacios mentales de la RIC anterior, en el cuerpo como control remoto, y permanece evidente al interactuar con el robot para producir su desplazamiento. La relación de propiedad se presenta en la coincidencia que tiene tanto el cuerpo por su propiedad de movimiento, como el robot con el sentido principal que se le designó de ser movido. Por último, la proyección reitera que para cada elemento existe un complemento, es decir, para cada movimiento del cuerpo existe una función equivalente. 
Retomando lo que se ha dicho en relación a que el significado construido a partir de la interacción con el robot, a través de una interface intangible, no es posible explicarse solamente con una RIC simple, sino que es necesario ayudarse de una red escópica que muestre mejor los elementos que intervienen en este ejercicio.

Para entender cómo el cuerpo se convierte en un control remoto, sin perder sus propiedades, es preciso observar cómo se produce el cambio del frame cultural, ausentando la existencia del control remoto real y dando paso al cuerpo humano. Para aclarar conviene considerar lo mencionado por Hutchins (2005):

Un modelo conceptual incorporado, expresado, asegurado se restringe entre elementos conceptuales. En ese orden juega un rol en el proceso de razonamiento, un modelo conceptual debe ser cognitivamente estable. Así, este debe mantener su sistema de restricciones mientras este sujeto a una manipulación mental o física. [...] Un modelo conceptual con estas propiedades es un modelo cultural. Con suficiente práctica, soporte y motivación un complejo y arbitrario mapeo puede ser aprendido. En un sistema de modelo cultural, cada modelo restringe y es restringido por una rica red de relaciones de otros modelos de sistemas de creencias culturales. Esto posibilita un razonamiento efectivo sobre problemas complejos cuando está expresado en términos de modelos culturales familiares. Parte del poder cognitivo de la metáfora deriva del efecto que es posible razonar efectivamente sobre conceptos no familiares, si ellos pueden primero ser amalgamados con conceptos familiares estables. (p. 1574)

Visto así, los anclajes materiales son modelos culturales que permiten el cambio de la relación con nuevos objetos o actividades y dan sustento a la investigación en términos del desempeño de los participantes al expresarse de conformidad con sus experiencias acerca de generar movimiento en un objeto.

\section{Hallazgos importantes de la experiencia}

Un aspecto importante que se encuentra en la experiencia vivida entre el robot móvil y las personas está relacionado con la manera de significar objetos nuevos a partir de la interacción con otros ya establecidos y definidos por una cultura. Es el caso del cambio que se dio en el reconocimiento de una nueva interfaz, llamada Kinect, para controlar el dispositivo; esto se logra a partir de romper el paradigma de manipulación asociado al trabajo con un joystick, que exige el contacto directo, contrarrestado con una tecnología que busca la total independencia del cuerpo de los objetos materiales, a través de la proyección de sus movimientos. Esta postura coincide con lo mencionado por Markussen y Krogh (2008): "el cambio de Frame cultural es lo que sucede en la mente de los usuarios cuando las nuevas experiencias de uso tecnológico impulsan a desarrollar estructuras de conocimiento" (p. 7).

Esta experiencia deja ver que hay un cambio a nivel de frame cultural suscitado por la modificación de la conducta de interacción en donde el cuerpo asume el rol del control remoto. En especial esto se evidencia en los niños, quienes no tenían un acercamiento previo a este dispositivo, lo cual no fue un impedimento para lograr movilizar el robot. Se observa que ellos se enfrentan a una reformulación de su espacio tridimensional para adaptar sus acciones en función de la programación del objeto, como se evidencia en que durante los primeros intentos de control, los movimientos se dieron en dos dimensiones: empujar y arrastrar; paulatinamente fueron modificándolos para adaptarse a la manera preconcebida de comandar el robot. 
Otro concepto importante en la TIC es el anclaje material, entendido como una estructura material suficientemente estable como para poder en actuarla, y sobre esta responsa información que se puede recuperar mediante kineto-percepción, lo cual permite tener una mejor "economía cognitiva" (Hutchins, 1995, citado por Niño, 2015, p. 553). En este estudio se evidenció que el surgimiento del "Cuerpo como control remoto" se sustenta en la existencia de un anclaje material como el "Control remoto", en donde se adoptan las propiedades de este mismo, relacionadas con la posibilidad de transmitir instrucciones a distancia y suprimir el contacto directo con la máquina, proyectado en el blend "[Mover robot con el cuerpo]2.

En ese mismo sentido, se reconoce que para poder diseñar un objeto nuevo que dé solución a un problema, se requiere de anclajes materiales que marquen la evolución de ese objeto, o incluso, que permitan crear uno nuevo, tal como se expuso en el ejemplo del portaminas. Para el caso en mención, la evolución del "Cuerpo como control remoto", solo es posible si no se desliga la concepción de un "Control remoto tangible", y este a su vez depende de la existencia previa de unos mandos localizados directamente en la máquina, enfatizando en que los anclajes materiales son importantes para la definición de nuevos objetos.

El éxito en el diseño de productos o la solución de problemas está relacionado con el rompimiento de paradigmas. La TIC puede resultar de especial utilidad en la consolidación de estos procesos, gracias a la posibilidad que tiene de explicar la construcción de estructuras cognitivas que integran diferentes conceptos, de distintos ámbitos y la proyección de estos para la construcción de nuevos significados que den origen a novedosas ideas, que dependen de la cercanía con los espacios mentales que circundan dicha solución.

El concepto de RICes mucho más amplio; no se limita a establecer relaciones de significado de un solo elemento, puesto que relaciona un sinnúmero de espacios mentales, como se explicó. De acuerdo con esta premisa, el proceso de construcción de redes más complejas es inherente al comportamiento humano; la profundización en el funcionamiento de la TIC puede contribuir a la formulación de nuevas metodologías de diseño.

Esta investigación culmina con las anteriores reflexiones, pero es nuestro interés dejar abiertas otras proyecciones de la TIC, en especial su relación con la educación. Algunas posibilidades contemplan la formulación de estrategias metodológicas, por ejemplo en la construcción de ambientes de aprendizaje en donde a partir de una problemática sea evidente que los estudiantes construyen sus propias RIC y llegan a la solución de un problema, enmarcado en el proceso que sustenta el funcionamiento de la TIC. En conclusión, lo que se pretende es llegar a la formulación de soluciones a través de la construcción de blends. Naturalmente, el diseño de esta metodología exigirá la comprensión por parte del maestro, de la manera como el estudiante relaciona conceptos, teje redes y genera significados. Esto por supuesto es útil en todos los campos del saber, 
y no exclusivamente con lo que tiene que ver con la enseñanza de la tecnología.

Los anclajes materiales pueden sustentar un aprendizaje más práctico, al permitir ligar conceptos propios de los elementos materiales con otros, para solucionar problemas. Allanando el camino para la formulación de metodologías alternativas que fortalezcan las didácticas de las diferentes disciplinas.

No obstante, en el campo de la educación en tecnología es importante indagar a la luz de la TIC sobre los procesos cognitivos que realizan las personas para programar un robot, en razón a que la experiencia demostró que la perspectiva que se tuvo para la programación del mismo no contempló las expectativas de los participantes, limitando las posibilidades de control. Esto permitiría validar los aportes de los estudiantes en un mejor desarrollo de algoritmos de programación que maximicen las opciones de control.

La tendencia tecnológica actual se encamina a maximizar la actividad corporal y la interacción con las máquinas a través de interfaces más naturales, suprimiendo incluso el contacto con los objetos y la creación y participación en mundos artificiales, es el caso de la realidad virtual y la realidad aumentada. La cercanía con estas tecnologías y la presencia de problemas comunes puede motivar su solución a través de la integración conceptual, siendo el Kinect o dispositivos similares, medios propicios para generar esos acercamientos.

\section{Referencias bibliográficas}

Barrio del Castillo, I.; González Jiménez, J.; Padín Moreno, L.; Peral Sánchez, P.; Sánchez Mohedano, I. y Tarín López, E. (2015). El estudio de casos.

Fauconnier, G. (1994). Mental space: Aspects of meaning construction in natural language. Nueva York: Cambridge University Press.

Fauconnier, G. and Turner, M. (2002). The way we think: Conceptual blending and the mind's hidden complexities. Nueva York: Basic Books.

Hutchins, E. (2005). Material anchors for conceptual blends. Journal of Pragmatics, 37, 1555-1577.

Joy, A.; Sherry, J. and Deschenes, J. (2007). Conceptual blending in advertising. Journal of Business Research, 62, 39-49.

Malagón, C. (2013). Intertextualidad, imágenes publicitarias e integración conceptual. En: D. Niño (ed.).Ensayos semióticos II (pp. 153-188). Bogotá: Universidad de Bogotá Jorge Tadeo Lozano.

Markussen, T. and Krogh, P. (2008). Mapping cultural frame shifting in interaction design with blending theory. International Journal of Design, 2, 5-17.

Niño, D. (2015). Elementos de semiótica agentiva. Bogotá: Universidad de Bogotá Jorge Tadeo Lozano.

Ruiz-Velasco, E. (2007). Educatrónica: innovación en el aprendizaje de las ciencias y la tecnología. Buenos Aires: Editorial Díaz de Santos. 